\title{
Controlled Growth of Dendrimer-Coated Gold Nanoparticles: A Solvent-Free Process in Mild Conditions
}

\author{
José Antonio Ulloa, Joaquín Barberá, and José Luis Serrano*
}

Cite This: ACS Omega 2021, 6, 348-357

Read Online

ABSTRACT: Monodisperse dendrimer-coated gold nanoparticles with a spherical shape have been obtained by direct reduction of $\mathrm{HAuCl}_{4}$ with sodium borohydride in the presence of dodecanethiol as a stabilizer and subsequent functionalization by ligand exchange reaction with polybenzylic thiolated dendrons. The substitution pattern of the dendrimeric units plays a fundamental role in the rate of the functionalization exchange process and consequently conditions the size and the polydispersity of the NPs obtained. An ulterior growth process occurs by thermal stimuli $\left(150{ }^{\circ} \mathrm{C}\right)$ in a solvent-free environment. This method, carried out in mild conditions, allows the formation of highly monodisperse gold NPs with different sizes for different time reactions, and we discuss the mechanisms involved in this process. Finally, we demonstrate the chemical composition and stability of our compounds by structural, thermal, and chemical characterization of the samples before and after thermal treatment.

\section{INTRODUCTION}

One of the objectives of the current research in nanoscience is the design of materials with novel properties that allow the creation of new devices or technologies. To achieve this goal, one of the most interesting approaches is the synthesis of metamaterials with nanometric dimensions because these compounds present new properties and different from those of the same materials on a macroscopic scale. The nanoparticle (NP) size reduction modifies the density of states, giving rise to new properties directly dependent on dimensions and shape $\mathrm{e}^{2,3}$ as it happens in the plasmonic ${ }^{4-7}$ or magnetic properties. ${ }^{8-10}$

In metal nanomaterials, the chemical nature of the surface plays a fundamental role. Frequently, these systems are stabilized by the functionalization of the external metallic nuclei with different types of molecular ligands. ${ }^{11}$ The final properties of this new multicomponent material are defined by the size and shape of the inorganic core and by the characteristics of the organic ligand and their interactions with the metallic surface. ${ }^{12,13}$ Among other substances, organic dendrimers appear as a powerful tool for the generation of new metallic-organic hybrid nanomaterials. ${ }^{14-25}$ Dendrimers are macromolecules with a well-defined chemical structure and allow the introduction of different active functions that can originate new physical and chemical properties in nanomaterials or improve properties already existing therein for applications in both biology ${ }^{26-30}$ and advanced materials. ${ }^{31-35}$

The applications of metal nanomaterials require careful control of some structural parameters. Thus, the distribution of the size, shape, and distance between the particles of the

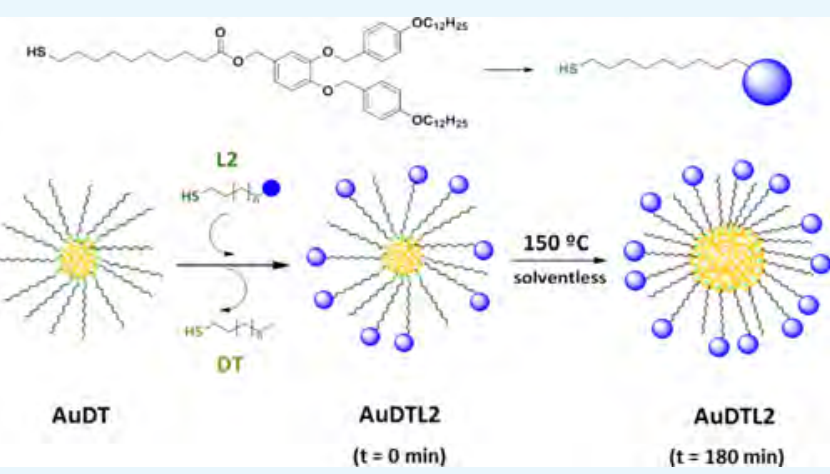

metallic nuclei conditions the physical properties of these nanomaterials. ${ }^{29-32}$ At present, there are two main techniques to control the growth of metallic nuclei: hot injection ${ }^{33-36}$ and the thermolysis process. ${ }^{37-40}$ In the first, metal salts dissolved in appropriate solvents are added at high temperature, while in the second, a thermal stimulus is applied to the organometallic precursory salt. Both methods present some problems that need to be controlled. Thereby, the extremely high temperatures of the heat treatment or the presence of the metal salt in the hot solution can lead to the aggregation or decomposition of the metallic core and the organic ligands, thus losing the homogeneity of the hybrid system. As a result, a more environmentally friendly approach to synthesis and control of metallic NPs becomes necessary. ${ }^{41-47}$

The crucial point in this process is the control of the NP size. It has been demonstrated that size distribution and steric crowding of the dendrimer surface determine the resulting behavior such as catalytic properties. ${ }^{48,49}$ The assembly of functional nanomaterials based on dendrimers can be controlled, for example, by ion selectivity. ${ }^{50}$

Received: September 22, 2020

Accepted: October 26, 2020

Published: December 22, 2020 
Scheme 1. Synthetic Pathway to the Thiol Focal Point Dendrons Bearing 3 (L3), 9 (L9), 2 (L2), and 4 (L4) Terminal Chains ${ }^{a}$

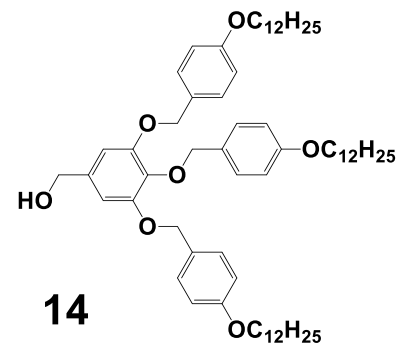

$\mid$

19

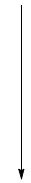

L3

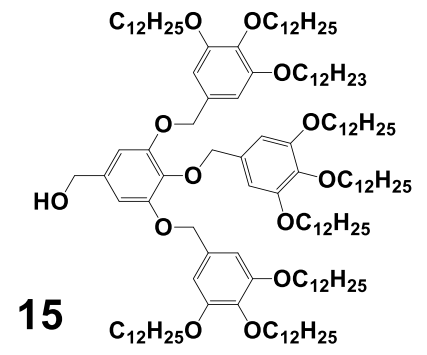<smiles>CCOc1ccc(COc2ccc(CO)cc2OCc2ccc(OCC)cc2)cc1</smiles>

16

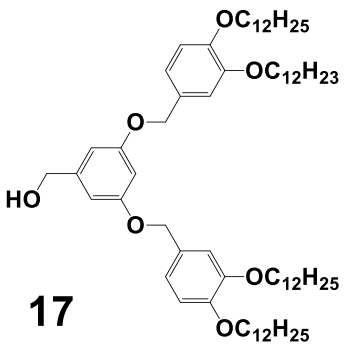

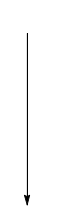

20<smiles>O=C(O)CCCCCCCCCCCCC(=O)O</smiles>

18

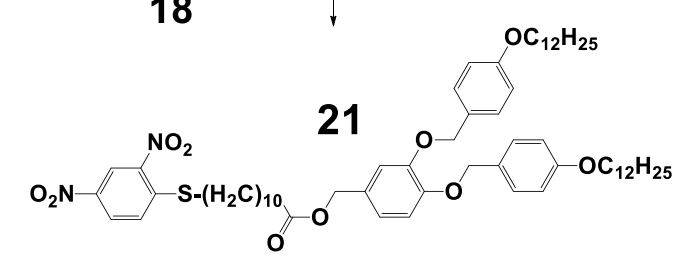

$\mathrm{HS}-\mathrm{CH}_{2}-\mathrm{CH}_{2}-\mathrm{OH}$

(TEA, DCM)

L9

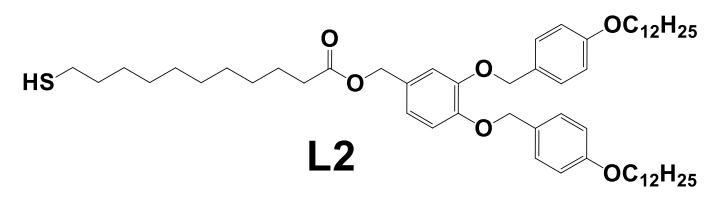

22

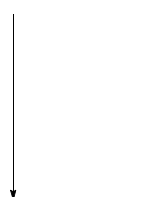

L4

${ }^{a}$ For simplicity, total structures are only drawn for compound L2. DCC: $N, N^{\prime}$-dicyclohexylcarbodiimide, DPTS: 4-(N,N-dimethylamino)pyridinium 4-toluenesulfonate, DCM: dichloromethane, TEA: triethylamine.

In this work, the size control is achieved by ligand exchange and thermal treatment. The synthesis of gold NPs coated with different Percec-type dendrons (dendrimer-coated gold nanoparticles, DenNP) by means of the ligand exchange method is presented. Subsequently, these NPs are subjected to a thermally controlled seeded-growth treatment at high temperatures for different times. The properties of the NPs before and after being subjected to thermal treatment are compared. Taking into account our previous experience in mesogenic dendrimers, we have chosen four different dendronic structures derived from 3,4-dihydroxy, 3,4,5-trihydroxy, and 3,5-dihydroxy benzyl alcohols. With the aim of simplifying the nomenclature, these dendronic structures are named L2, L3, L9, and L4, indicating the number of terminal chains incorporated in each dendronic structure (see Scheme 1).

Three of these dendrons bearing, respectively, two, three, and nine dodecyloxy terminal chains have been proved to favor the mesogenic behavior. On the other hand, the fourth dendron, which has a 3,5-substituted structure with four dodecyloxy end chains, does not favor the appearance of liquid crystal properties. $^{51-55}$

All the dendrons are functionalized with a 11-mercaptoundecanoate group in the focal point in order to incorporate a terminal thiol group, capable to interact with the gold NPs, and a long flexible chain that favors the distribution of the dendrons on their surface and minimizes the steric difficulties for the dendrons to approach.
The DenNPs were obtained by a two-step process. In a first step, the gold NPs were synthesized by the modified BrustSchiffrin method, with 1-dodecanethiol (DT) as a stabilizer. In the second step, the NP stabilized with DT was treated in a dichloromethane (DCM) solution with different dendrons functionalized with a thiol group at their focal point, which results in a partial exchange with the ligands of DT and incorporation of new molecules to available gold atoms on the surface. These hybrid NPs were subjected to a heat treatment at $150{ }^{\circ} \mathrm{C}$ in a solvent-free environment for $30,60,120$, and 180 min. Finally, the modulation of the size and distribution of the NPs obtained after the thermal process as well as the mechanism thereof were studied. The size and morphology of all the NPs were determined at each stage by transmission electron microscopy (TEM) and their chemical nature was investigated in depth by a variety of techniques.

\section{MATERIALS AND METHODS}

The materials and equipment used in the preparation and characterization of the compounds described in this paper are gathered in Section 1 of the Supporting Information.

The synthesis of the precursory Frechet-like polybenzyl alcohol dendrons was described previously. ${ }^{56,57}$ The synthesis Schemes S1 and S2, the experimental procedure, and the characterization of the four polybenzyl alcohols synthesized are gathered in Section 2.1 of the Supporting Information.

The synthesis of the dendritic thiols L2, L3, L4, and L9 is described in Scheme 1. All the compounds were characterized 
by nuclear magnetic resonance $\left({ }^{1} \mathrm{H}\right.$ NMR and $\left.{ }^{13} \mathrm{C} \mathrm{NMR}\right)$, Fourier-transform infrared spectroscopy (FTIR), and mass spectrometry (EM-MALDI). The details of the synthesis and characterization of all the functionalized dendrons with the thiol group at the focal point are described in detail in Sections 2.1.4 and 2.1.5 of the Supporting Information. As a representative example that demonstrates the formation of the thiol focal point dendron and the cleavage of the protecting group, the ${ }^{1} \mathrm{H}$ and ${ }^{13} \mathrm{C}$ NMR spectra and the MS-MALDI spectra of $\mathbf{L} 2$ and its protected precursor $\mathbf{2 1}$ are shown and discussed in Figures S1 and S2 of the Supporting Information.

The experimental procedure for the preparation of the gold dendrimeric NPs is described in Section 2.2.1 of the Supporting Information. Precursory gold NPs stabilized with DT (AuDT) were synthesized by the method of Brust et al. ${ }^{58}$ by direct reduction of $\mathrm{HAuCl}_{4}$ with sodium borohydride in the presence of dodecanethiol (DT) as a stabilizer and tetrabutylammonium bromide (BTBA) as a transfer agent in a biphasic system (toluene and Milli-Q water). The change in coloration of the dispersion from yellow to dark red indicates the formation of NPs. The isolated NPs were submitted to a ligand exchange reaction with each of the dendritic thiols L2, L3, L4, and L9, following the method described by Murray et al. (Figure 1). ${ }^{59}$

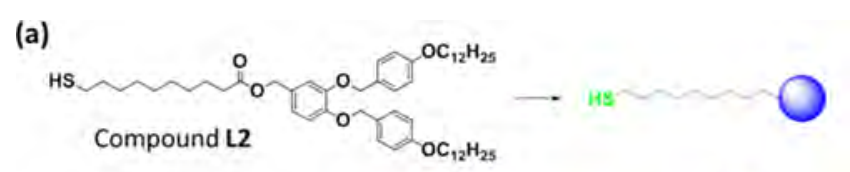

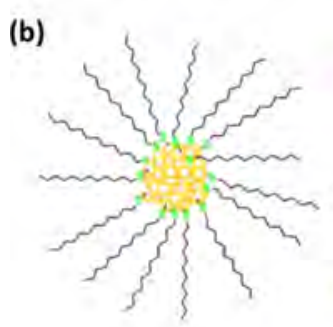

AuDT

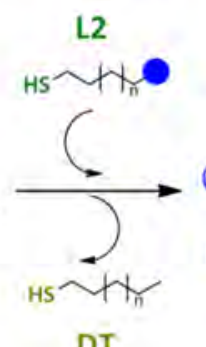

DT

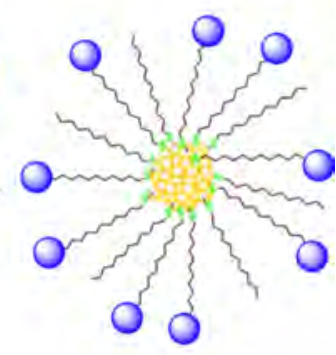

AUDTL2
Figure 1. (a) Thiol focal point dendron L2, chemical structure and schematic representation of compound $\mathbf{L} \mathbf{2}$ as an example, and (b) schematic representation of the ligand exchange reaction.

For a simple nomenclature of the samples, the gold NPs stabilized with DT will be denoted as AuDT and the hybrid NPs as AuDTLn.

The NPs were purified by size exclusion chromatography (SEC), yielding in all cases a black solid. The SEC technique ensures that the free thiols have been removed and only the thiols bonded to the NP remain.

NPs were characterized by proton nuclear magnetic resonance ( ${ }^{1} \mathrm{H}$ NMR) (see Section 2.2.2 of the Supporting Information, Figures S3-S7) and X-ray photoelectron spectroscopy (XPS) (see Table S2 in Section 2.2.3 of the Supporting Information). Their thermal properties were studied by optical polarizing microscopy, thermogravimetric analysis (TGA), and differential scanning calorimetry (DSC) (see Table S3 in Section 2.2.4 of the Supporting Information). The NP size distribution was determined by scanning TEM (STEM) (see Section 2.2.5 of the Supporting Information).

\section{RESULTS AND DISCUSSION}

The benzyl alcohol precursors (see Scheme 1) show liquid crystal behavior. Compounds 15 and 16 exhibit enantiotropic mesogenic behavior, showing cubic and hexagonal columnar mesomorphism, respectively. On the other hand, compounds 14 and $\mathbf{1 7}$ show only a monotropic columnar hexagonal phase. The observed temperature transitions agree with the data described in the literature (see Table S1 in Section 2.1.3 of the Supporting Information). ${ }^{32,51-55}$

The ligand exchange process used in this work was successful with the selected dendritic thiols (compounds L2, L3, L4, and L9).

Other approaches such as the direct reduction of the gold salt to produce NPs in the presence of the dendritic ligands result in macroscopic aggregates and polydisperse systems. The methodology that we use in this research guarantees a good degree of functionalization, size and shape monodispersity for the AuNP and reproducibility of the results obtained. This is a clear advantage over other methods such as direct reduction of the gold salt in the presence of dendrons, which often results in macroscopic aggregates.

It is possible to verify the ligand exchange process by ${ }^{1} \mathrm{H}$ NMR spectroscopy. As an example, in Figure 2, left side, it is possible to observe the incorporation of the signals because of ligand $\mathbf{L 9}$ (c) in the AuDT template (b), giving place to the hybrid system AuDTL9 (a). In the same way, we can observe the shift of the $c_{1}$ triplet signal at $2.50 \mathrm{ppm}$ in $\mathbf{L 9}$ (corresponding to the neighboring $\mathrm{CH}_{2}$ to the $\mathrm{SH}$ group $-\mathrm{CH}_{2} \mathrm{CH}_{2} \mathrm{SH}$ ) to higher values in the multiplet signal $c_{1-2}$ found for AuDTL9 $\left[-\left(\mathrm{CH}_{2}\right)_{9}-\mathrm{CH}_{2}-\mathrm{SAu}\right]$ at $2.67 \mathrm{ppm}$ when the ligand is incorporated to the AuNP.

The XPS studies demonstrated that no aggregation or decomposition of the particles occurred after the ligand exchange reaction, as only the characteristic doublet peaks of gold $4 \mathrm{f}_{7 / 2}$ and $4 \mathrm{f}_{5 / 2}$ orbitals with values close to 83.7 and $87.4 \mathrm{eV}$ and the complex signal of the sulfur $2 \mathrm{p}_{3 / 2}$ and $2 \mathrm{p}_{1 / 2}$ orbitals at 162.6 and $163.7 \mathrm{eV}$ were observed (Figure 2f,g and Table S2 in Section 2.2.3 of the Supporting Information).

The thermal properties of the NPs and dendritic thiols were studied by polarizing optical microscopy, DSC, and TGA to examine the thermal stability and the possible occurrence of liquid crystal phases when the samples are submitted to a thermal cycle on a heating stage (See Table S3 in Section 2.2.4 of the Supporting Information). Neither the pure dendritic thiols, L2, L3, L4, and L9, nor the derived NPs AuDTLn presented mesomorphism. By DSC, a direct transition from a crystalline phase to an isotropic liquid was observed for all the AuDTLn NPs. TGA studies are particularly relevant in the case of NPs because this technique allows an easy quantification of the relative proportions of organic and inorganic matter in the sample. These studies were performed under the air atmosphere from room temperature up to $800{ }^{\circ} \mathrm{C}$. In these conditions, the remaining mass at the end of the experiment corresponds exclusively to the inorganic fraction, in this case metallic gold (Figure 3). It is observed that all samples are stable up to temperatures above $200{ }^{\circ} \mathrm{C}$ and the percentage of gold decreases as the size of the dendritic ligand increases (see Table 1).

When the dendritic thiols have been incorporated by ligand exchange, the size and nearly spherical shape of the particles remain practically constant for AuDTL2, AuDTL3, and AuDTL9, compared to AuDT, as has been observed by TEM in the scanning mode, STEM (see Table 1, Figures 2d,e, and S8 

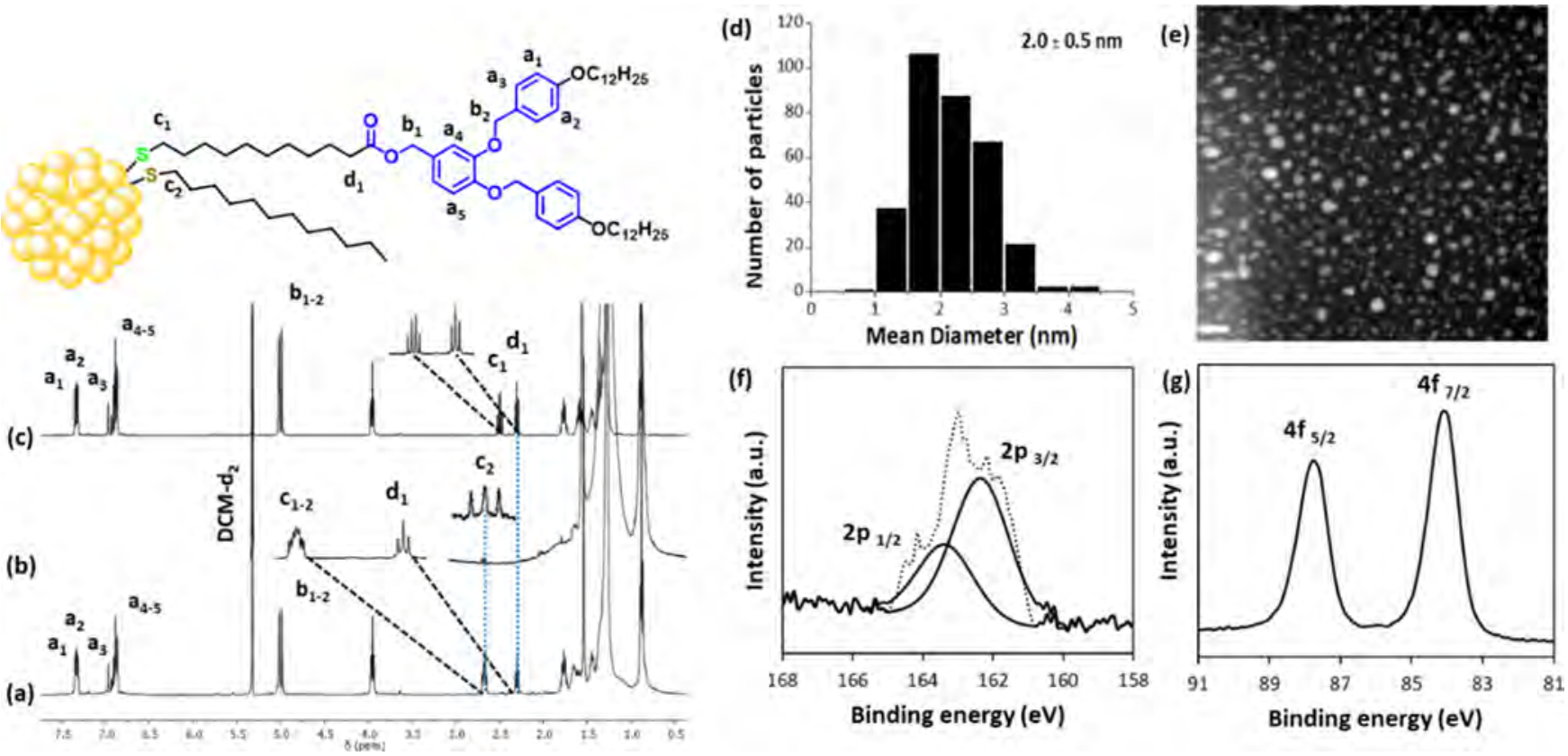

Figure 2. ${ }^{1} \mathrm{H}$ NMR (left) spectra in solution at $25^{\circ} \mathrm{C}$ of (a) hybrid AuDTL2, (b) AuDT template, and (c) L2 ligand. TEM results of AuDTL2: (d) NP size distribution histogram and (e) TEM micrograph. XPS spectra of AuDTL2. (f) S 2p spectrum and (g) Au 4f spectrum.

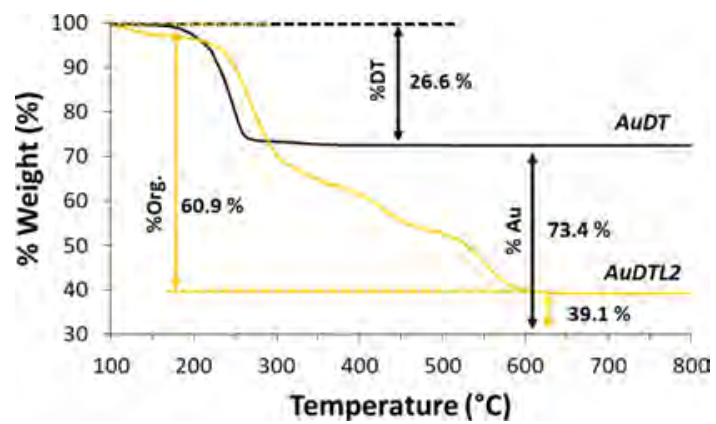

Figure 3. TGA graph of weight changes as a function of the temperature for AuDT and AuDTL2.

in Section 2.2.5 of the Supporting Information). On the other hand, a higher size is observed for AuDTL4, which contains NPs with a mean diameter of $2.7 \pm 0.6 \mathrm{~nm}$. These data confirm that in the ligand exchange process with ligand $\mathbf{L} 4$ a redistribution of the gold atoms and the ligands occurs. It is important to remember that the L4 ligand has a 3,5 disubstituted and their steric requirements are different from those of the other ligands L2, L3, and L9, which have a wedge shape. After measuring the mean diameters by STEM, it is possible to estimate the mean number of gold atoms per particle $\boldsymbol{n}_{\mathrm{Aw}}$, assuming that, at a nanoscopic scale, gold crystallizes in a face-centered cubic (fcc) system (confirmed by us by electron diffraction, see below) and the NPs are spherical (see Section 2.2.6 in the Supporting Information). The mean number of gold atoms per particle $\boldsymbol{n}_{\mathrm{Au}}$ deduced from this equation is gathered in Table 1.

An estimation of the percentage of each type of ligand (DT and $\mathbf{L} n$ ) stabilizing the NPs can be made by ${ }^{1} \mathrm{H}$ NMR spectroscopy. For this study, we have selected signals $\mathbf{d}_{1}$ and $\mathbf{c}_{1-2}$ because both signals appear isolated in the spectra of all dendronic NPs and allow an appropriate integration (see Figures 4 and S3-S7 in Section 2.2.2 of the Supporting Information).

The triplet signal $\mathbf{d}_{\mathbf{1}}$ is specific of the dendronic ligand $(\mathbf{L} \boldsymbol{n})$ and corresponds to the methylene group attached to the carbonylic group. Signals $\mathbf{c}_{1}$ and $\mathbf{c}_{2}$ are overlapped and correspond to the methylene hydrogens in alpha position to sulfur $\left(\mathrm{AuS}-\mathrm{CH}_{2}-\right)$ of dendron $\mathbf{L} \boldsymbol{n}$ and dodecanethiol DT, respectively. As an example, for AuDTL2 (see Figure 4) signal $\mathrm{d}_{1}$ appears at $2.3 \mathrm{ppm}(\mathrm{t}, 2 \mathrm{H})$, whereas signals $\mathrm{c}_{1-2}$ appear at 2.6-2.7 ppm. To calculate the proportion of each ligand, the

Table 1. Size and Composition of the Average NPs: Mean Diameter $(\Phi)$, Statistical Dispersity (SD), Calculated Number of Gold Atoms $\left(n_{\mathrm{Au}}\right)$, Weight Percentage of the Gold Part (Au\% Weight), Calculated Weight of the Gold Part, Calculated Weight of the Organic Part, Percentage of Dodecanethiol Ligand DT (\% molar), and Percentage of Dendrimeric Ligands Ln (\% Molar)

\begin{tabular}{|c|c|c|c|c|c|c|c|c|}
\hline sample & $\Phi^{a}(\mathrm{~nm})$ & $\mathrm{SD}^{a}(\mathrm{~nm})$ & $n_{\mathrm{Au}}{ }^{b} n$ gold atoms & $\% \mathrm{Au}^{c}$ (\% weight) & $\mathrm{Au}^{d}$ (Daltons) & $\operatorname{organic}^{d}$ (Daltons) & $\% \mathbf{D T}^{e}$ (\% molar) & $\% \mathbf{L} n^{e}$ (\% molar) \\
\hline AuDT & 2.0 & 0.5 & 247 & 73.4 & 48,700 & 17,700 & 100 & 0 \\
\hline AuDTL2 & 2.0 & 0.5 & 247 & 40.2 & 48,700 & 72,400 & 34 & 66 \\
\hline AuDTL3 & 2.2 & 0.5 & 329 & 39.1 & 65,800 & 101,000 & 53 & 47 \\
\hline AuDTL4 & 2.7 & 0.6 & 608 & 33.8 & 119,800 & 234,600 & 41 & 59 \\
\hline AuDTL9 & 2.0 & 0.5 & 247 & 22.1 & 48,700 & 171,500 & 33 & 67 \\
\hline
\end{tabular}

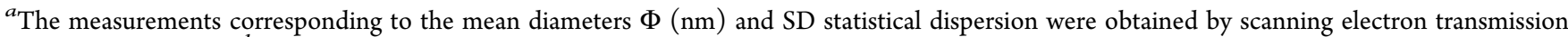
microscopy (STEM). ${ }^{b}$ The number of gold atoms per particle was calculated by Liu equation. ${ }^{c_{\%}} \%$ weight of the gold part in the hybrid NP was obtained by TGA. ${ }^{d}$ Calculated weight of the Au part and the organic part. ${ }^{e}$ The proportion of each ligand [\% DT (dodecanethiol) and $\%$ Ln (dendronic ligand)] (\% molar) was obtained by means of a one-dimensional quantitative experiment of $1 \mathrm{H}$ NMR. 


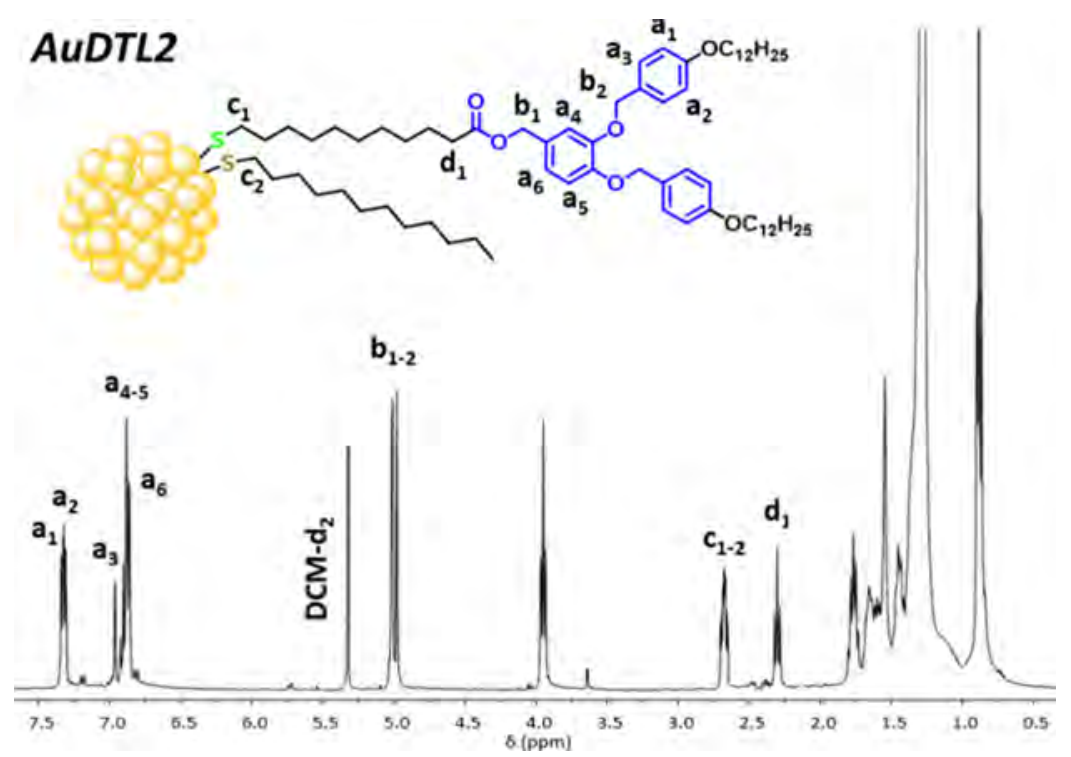

Figure 4. ${ }^{1} \mathrm{H}$ NMR spectrum of AuDTL2 in DCM- $d_{2}$ as a solvent.

area under signal $\mathbf{d}_{1}$ was measured, that corresponds to $2 \mathrm{H}$ of the dendron and should be equivalent to the area under signal $c_{1}$ of the same ligand $\mathbf{L} 2$. Now, the combined area for the methylene protons in alpha to the sulfur atom $c_{1}$ and $c_{2}\left(\mathrm{AuS}-\mathrm{CH}_{2}-\right)$ corresponds to the sum of the contributions of both ligands. As the contribution of signal $c_{1}$ must be equivalent to the value obtained for the area of signal $\mathbf{d}_{1}$ of ligand $\mathbf{L} 2$, the contribution of protons $\mathbf{c}_{2}$ of DT can be estimated. Using these data, the molar percentage of the two ligands could be easily calculated. The procedure is detailed in the Supporting Information (see Section 2.2.7). The resulting ligand molar proportions for all the NPs are gathered in Table 1.

The analysis of the data in Table 1 allows us to obtain relevant information about the ligand exchange process in these gold NPs. Besides the information about the NP size commented previously, the calculated number of gold atoms and the weight percentage of the inorganic part (Au content) obtained by TGA measurements allow to obtain the weight of inorganic and organic parts in an average NP. Thus, the weight of the gold part in Daltons is obtained multiplying the number of atoms in each NP by the atomic weight of the gold (196.96) and the organic part weight is obtained on the basis of each ligand percentage. These data together with the molar percentage of the ligands obtained by ${ }^{1} \mathrm{H}$ NMR spectroscopy allow obtaining the percentage of each type of ligand in the average NP. It is important to take into account that these data are approximate because of the intrinsic error of the techniques used (e.g., the estimated error for the NMR technique is $3 \%$ ); however, the information obtained is very useful to understand and explain the experimental results. As can be seen in Table 1, the ligand exchange process in the NPs is not total. The remaining percentage of dodecanethiol ligand (DT) is very similar for the gold NPs AuDTL2 (34\%) and AuDTL9 (33\%). The other two NPs, AuDTL3 and AuDTL4, show an increase in diameter, and in both cases a bigger percentage of dodecanethiol ligand remains (53 and $41 \%$, respectively). The change observed in these latter NPs suggests that the process of ligand exchange is slightly different from the process for ligands L2 and L9.

Thermally Tunable NPs: Growth Process of Gold NPs. The importance of the control on the size, shape, and dispersity for the applications of NPs was mentioned above. In this work, a procedure has been used to employ the AuNPs as seeds for their ulterior growth under controlled conditions. Other processes such as the heat treatment of organometallic salts involve significant amounts of thermal energy (even up to the calcination of the ligands), which produces variability and lack of reproducibility in the sizes of the NP obtained. On the other hand, the hot-injection of reagent involves a solution at a boiling temperature at which precursor metal salts are progressively added. Although the NP size distributions are better controlled, the use of salt solutions in different types of solvents does not result in such an environmentally friendly process. Our procedure is based on a one-pot method carried out in mild conditions in the absence of a solvent. To this end, the NPs containing all the types of dendritic thiols investigated were thermally treated. This treatment produces a quasisolid state that allows NP growth and avoids aggregate formation or decomposition phenomena. Moreover the absence of solvent or any other reactant guaranties the purity of the process. In this process, the temperature and the time are crucial to control the growth rate and the stability of the obtained material. In the thermal characterization section (see Table S3 in Section 2.2.4 of the Supporting Information), it was established that the decomposition of the NPs begins, in general, above $200{ }^{\circ} \mathrm{C}\left(T_{5 \%}\right.$ $>215{ }^{\circ} \mathrm{C}$ for all NPs), whereas melting points (crystal-toisotropic liquid transition) are in all cases below $100^{\circ} \mathrm{C}$. On this basis, an intermediate temperature value of $150{ }^{\circ} \mathrm{C}$ was chosen for the thermal treatment because at that temperature the materials are in a fluid state and at the same time thermal decomposition is avoided. The chosen temperature also ensures an adequate process rate to differentiate the successive steps of nucleation and progressive growth after variable reaction times $(15,30,60,120$, and $180 \mathrm{~min})$. The process was carried out on several batches of each sample that were removed from the thermal stimulus after the corresponding treatment time. After thermal treatment, the NPs were submitted to a purification process that includes washing, extraction, and SEC.

All the processed NPs were evaluated by XPS (see Table S4 in Section 3.1 of the Supporting Information), TGA (see Table S5 in Section 3.2 of the Supporting Information), one-dimensional quantitative ${ }^{1} \mathrm{H}$ NMR (see Table $\mathrm{S} 6$ in Section 3.3 of the Supporting Information), and TEM using the same method- 
Table 2. TEM and UV-Visible Spectroscopy Data of the AuDT and AuDTLn NPs after Isothermal Treatment at $150{ }^{\circ} \mathrm{C}$

\begin{tabular}{|c|c|c|c|c|c|c|c|c|c|}
\hline \multirow[t]{2}{*}{ Sample } & \multirow[b]{2}{*}{ time $^{a} \min$} & \multirow{2}{*}{$\frac{\text { AuDT }}{\Phi^{b}(\mathrm{DS})^{c}(\mathrm{~nm})}$} & \multicolumn{2}{|c|}{ AuDTL2 } & \multicolumn{2}{|c|}{ AuDTL3 } & \multicolumn{2}{|c|}{ AuDTL4 } & \multirow{2}{*}{$\frac{\text { AuDTL9 }}{\Phi^{b}(\mathrm{DS})^{c}(\mathrm{~nm})}$} \\
\hline & & & $\Phi^{b}(\mathrm{DS})^{c}(\mathrm{~nm})$ & $\lambda_{\max }^{d}(\mathrm{~nm})$ & $\Phi^{b}(\mathrm{DS})^{c}(\mathrm{~nm})$ & $\lambda_{\max }^{d}(\mathrm{~nm})$ & $\Phi^{b}(\mathrm{DS})^{c}(\mathrm{~nm})$ & $\lambda_{\max }^{d}(\mathrm{~nm})$ & \\
\hline & 0 & $2.0(0.5)$ & $2.0(0.5)$ & & $2.2(0.5)$ & & $2.7(0.6)$ & & $2.0(0.5)$ \\
\hline & 15 & $1.8(0.8)$ & $2.2(1.5)$ & & $2.4(0.7)$ & 512 & $5.1(1.2)$ & 528 & $2.2(0.4)$ \\
\hline & 30 & $2.3(0.8)$ & $2.9(1.3)$ & & $2.7(0.5)$ & 512 & $7.6(0.5)$ & 530 & $2.4(0.4)$ \\
\hline & 60 & $2.8(1.0)$ & $3.5(0.8)$ & 509 & $3.6(0.5)$ & 517 & $7.7(0.6)$ & 531 & $2.4(0.4)$ \\
\hline & 120 & $3.1(0.6)$ & $4.9(0.5)$ & 526 & $4.1(0.5)$ & 523 & $8.1(0.7)$ & 533 & $2.6(0.5)$ \\
\hline & 180 & $3.2(0.8)$ & $5.1(0.5)$ & 529 & $4.6(0.6)$ & 527 & $8.3(0.6)$ & 535 & $2.6(1.5)$ \\
\hline
\end{tabular}

${ }^{a}$ Time of treatment $(\mathrm{min}) .{ }^{b}$ Mean diameter $\Phi .{ }^{c}$ Statistical dispersion SD (nm). ${ }^{d}$ Max. wavelength of the resonance surface plasmon (RSP) band when observed. These values were obtained by measurements of the microphotographs with the image software package.
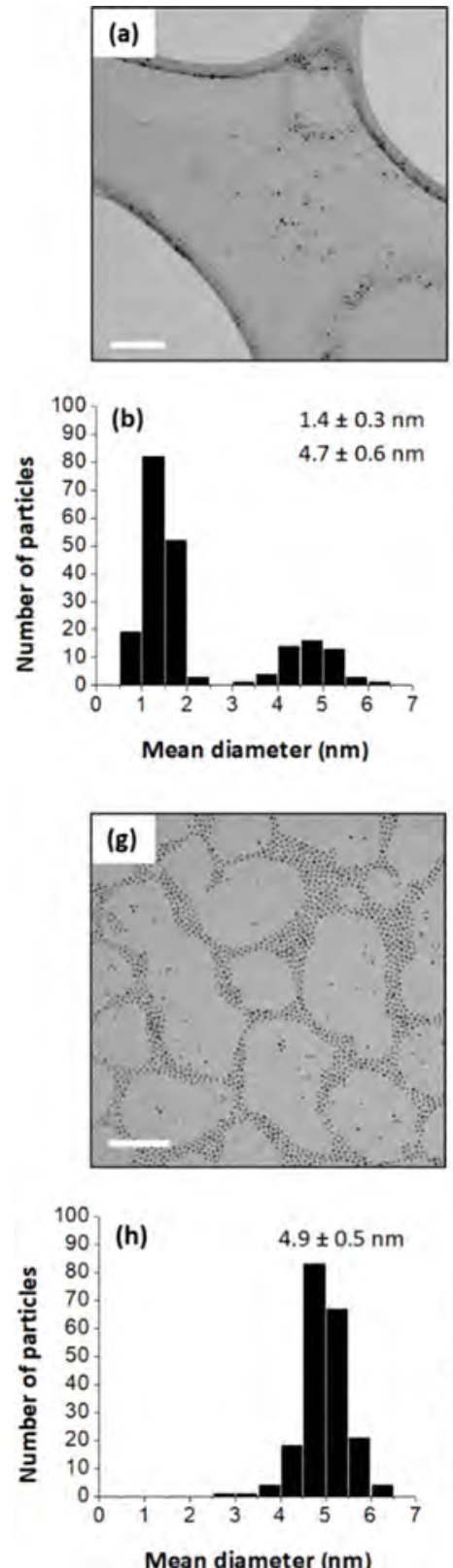
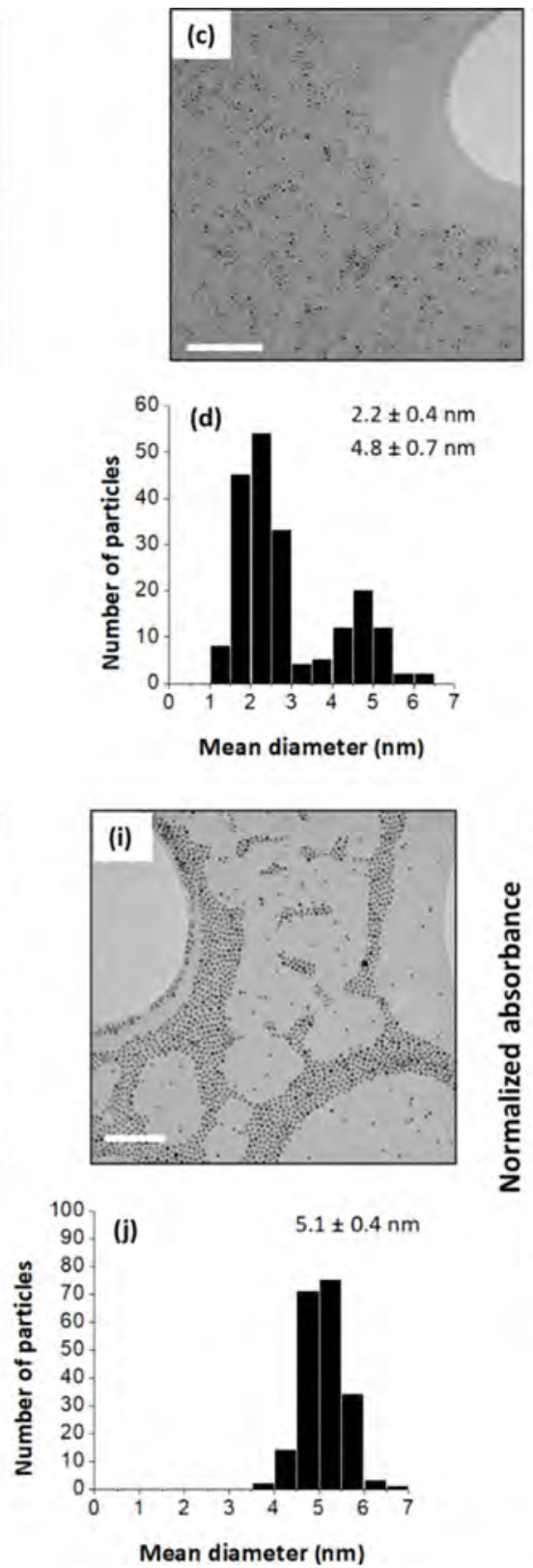
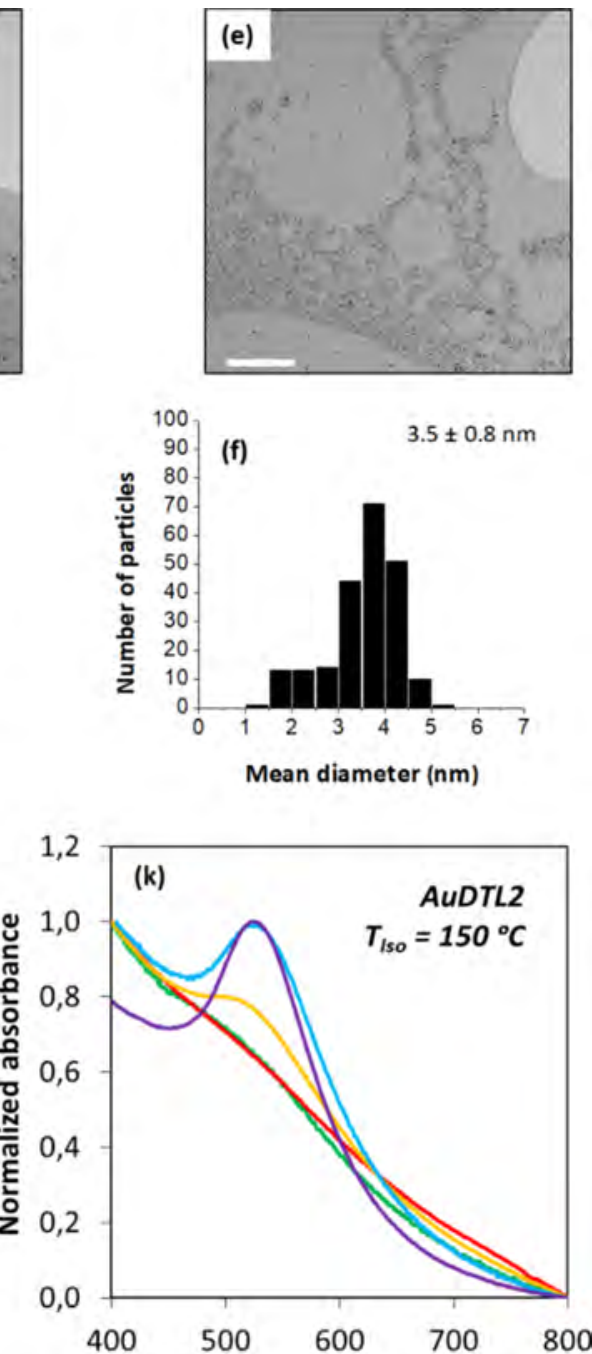

Wavelength $(\mathrm{nm})$

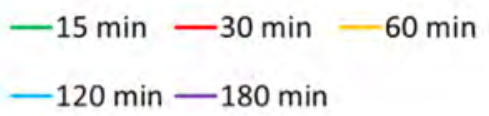

Figure 5. TEM microphotographs (a,c,e,g,i) and NP size distribution histogram (b,d,f,h,j) of AuDTL2 after 15, 30, 60, 120, and 180 min of isothermal treatment and (k) UV-vis spectra of AuDTL2 NPs in DCM solution after the isothermal treatment.

ology as that for the precursory NPs (see Table 2). It is important to emphasize that XPS and TGA studies were carried out on the raw material obtained after the growth processes because our main interest is to know the changes in NP size and shape. Only ${ }^{1} \mathrm{H}$ NMR studies of the NPs were performed in solution, after removing excess ligands that are not bound to the gold NPs. 
These techniques probe that the treated NPs do not show significant differences with the starting systems. This fact confirms that the growth treatment does not generate decomposition processes neither in the ligands nor in the gold NPs. Thus, the XPS signals can be used to find out if the thermal tuning of the samples has occurred without generating subproducts or decomposition of the starting material. In Table S4, it can be observed that for all the samples the highresolution signals characteristic of the $\mathrm{S} 2 \mathrm{p}$ and $\mathrm{Au} 4 \mathrm{f}$ orbitals do not change compared to the samples before thermal treatment $(t$ $=0 \mathrm{~min}$ ). This result indicates that the isothermal process does not cause decomposition phenomena, such as gold or sulfur oxidation, which would modify multiplicity (from a doublet to a singlet for $\mathrm{Au} 4 \mathrm{f}$ upon going from $\mathrm{Au0}$ to $\mathrm{AuI}$ ) or the signal values in electronvolt. TGA studies (Table S5) of the tuned NPs were made in an air atmosphere from room temperature to 800 ${ }^{\circ} \mathrm{C}$ at a heating rate of $10{ }^{\circ} \mathrm{C} \mathrm{min}^{-1}$. Both the temperatures at which mass losses and total mass loss occur are similar before and after the NP treatment. All samples show a thermal stability similar to that of pristine NPs. Finally, the ${ }^{1} \mathrm{H}$ NMR data of the thermally treated NPs show that, after isothermal treatment, the ratios of DT and $\mathbf{L} n$ ligands in the NPs do not change significantly (see Table S6). In Figures S10-S13 (Section 3.3 of the Supporting Information), the evolution of the ${ }^{1} \mathrm{H}$ NMR spectra of the NPs after heat treatment has been represented.

The results of the TEM studies are especially relevant and provide outstanding information on the effects of isothermal treatment on the NP size distribution. As can be seen in Table 2, all the samples show NP size growth as the isothermal treatment progresses. Figure 5 shows the TEM image and the size distribution for AuDTL2 NPs after 15, 30, 60, 120, and $180 \mathrm{~min}$ of thermal treatment. It is important to note that for each TEM specimen, 200-250 particles at least have been measured from different microphotographs from different areas of the grid.

Before treatment, the mean size of these NPs was $2.0 \pm 0.5$ $\mathrm{nm}$. After $15 \mathrm{~min}$ of isothermal treatment at $150{ }^{\circ} \mathrm{C}$, a mean diameter $2.2 \pm 1.5 \mathrm{~nm}$ and a bimodal distribution are obtained: a fraction with $1.4 \pm 0.3 \mathrm{~nm}$ ( $74 \%$ of NPs in the sample) and another fraction with $4.8 \pm 0.6 \mathrm{~nm}$ ( $26 \%$ of NPs in the sample). The thermal process that generates NPs with smaller sizes than the starting NPs is named thermolysis. This process initially generates metallic nuclei that act as seeds and begin to grow through slow diffusion of the metal atoms through the organic medium or through mutual interaction between different seeds. When the reaction time is increased to $30 \mathrm{~min}$, a bimodal size distribution is still observed $(2.2 \pm 0.4 \mathrm{~nm}$ for $74 \%$ and $4.8 \pm 0.7$ $\mathrm{nm}$ for $26 \%$ of the AuNPs). However, the mean diameter is larger $(2.9 \pm 1.3 \mathrm{~nm})$ and the dispersion tends to decrease. After reaction times of 60,120 , and $180 \mathrm{~min}$, the AuNPs keep growing in diameter and in size and shape homogeneity to $3.5 \pm 0.8,4.9$ \pm 0.5 , and $5.1 \pm 0.4 \mathrm{~nm}$, respectively. The data gathered in Table 2 and shown in Figure 5 suggest that the isothermal treatment at $150{ }^{\circ} \mathrm{C}$ of AuDTLn samples for different time intervals produces three processes: (a) initial thermolysis of the metal nuclei to form seeds (thermal shock); (b) diffusion of the gold atoms or larger metal seeds through the organic medium to produce the progressive and homogeneous growth of the NPs, and (c) Ostwald ripening, a phenomenon in which the smaller NPs associate to generate bigger NPs. A schematic representation of this stepwise process is drawn in Figure 6. At the same time, the size dispersion decreases as the thermal treatment progresses, which implies that not only growth but also size and shape monodispersity is achieved.
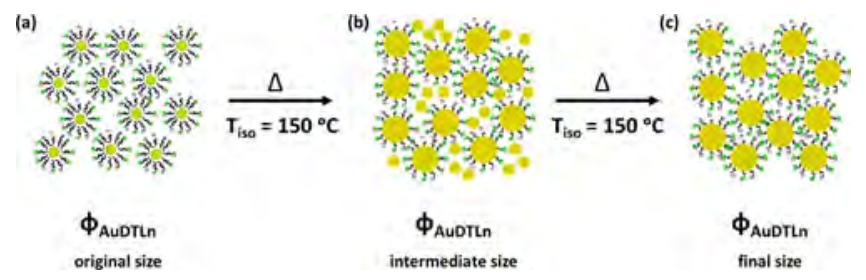

Figure 6. Schematic representation of the mechanism of seeding and growth of metallic AuNPs by the effect of temperature $\left(T_{\text {iso }}\right)$. (a) Initially, the AuNPs are subjected to thermal shock for 15-30 min; this process gives rise to the stage (b), where homogeneous growth of AuNP occurs by diffusion of the gold seeds (small particles) through the dendritic matrix (Ln), giving rise to large AuNP. (c) Finally, the homogeneous growth of the NPs stops after consuming all the gold seeds and then Ostwald ripening is the only possible processes.

The seed formation [stage (a)] takes the first 15 min and then growth and association of the AuNPs by slow diffusion through the dendritic matrix [stage (b)] take place. The growth rate and the mean sizes depend on the dendron type and the number of peripheral chains and not on the total reaction time or the temperature, which are the same for all the systems under study. Finally, in a final saturation stage [stage (c)], the NPs do not grow any longer and a homogeneous size distribution is reached. A graphical representation of the continuous growth process of the Au NPs is shown in Figure 7.

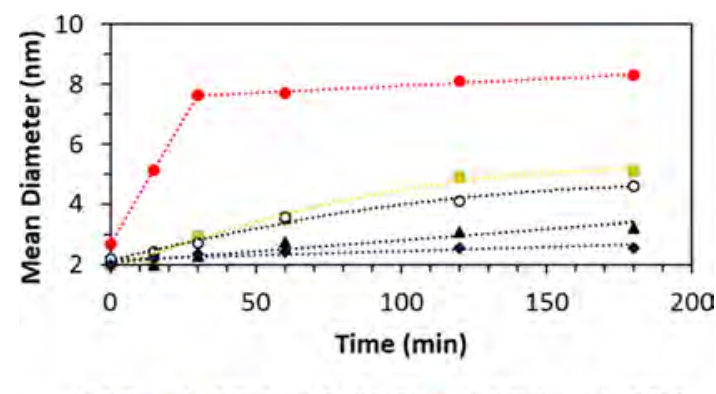

$\triangle$ AuDT $=$ AuDTL2 OAuDTL3 - AuDTL4 - AuDTL9

Figure 7. Graphical representation of the continuous growth process of AuNPs (mean diameter in $\mathrm{nm}$ ) by an applied thermal stimulus after different reaction times (in minutes) for all the samples. In each TEM specimen, 200-250 particles at least have been measured from different microphotographs obtained from different areas of the grid.

This representation can help to interpret the results obtained. From the data in Table 2 and Figure 7, it is concluded that there are two regimes in the growth of the AuNPs: (a) for AuDT, AuDTL2, AuDTL3, and AuDTL9, the evolution of the mean diameter shows a similar trend as a function of the reaction time. In all cases, a progressive NP growth is observed, the rate of which decreases as the time increases. The growth is smooth for AuDT and AuDTL9 and more pronounced for AuDTL2 and AuDTL3. In the case of AuDTL9, probably the congested surface of the NP (there is a big number of dendronic ligand molecules bearing nine terminal chains) renders the thermolysis process difficult and, consequently, hinders the formation of gold seeds and the growth process. A lower number of hydrocarbon chains in the dendron periphery favor the metal nuclei growth. The low growth of the AuDT particles can be attributed to the lower NP stabilization with the DT ligand than with the dendronic ligands. As homogeneity is concerned, the materials become more monodisperse upon thermal treatment until a point (around $120 \mathrm{~min}$ ), in which growth stops because 
of the lack of seeds and the NPs begin to associate into bigger NPs (Ostwald ripening). ${ }^{60-62}$ This is particularly evident for AuDTL9 at $180 \mathrm{~min}$, which goes from a dispersity of 0.5 at 120 min to 1.5 at $180 \mathrm{~min}$. As can be seen in Table 2, the best results (in growth and homogeneity) of the thermal treatment for compounds AuDTL2, AuDTL3, AuDTL9, and AuDT are obtained for times near $120 \mathrm{~min}$. On the other hand, for AuDTL4, the growth is faster and greater, so that the highest values are practically reached already at 15-30 min (Figure 7). As was mentioned above, the dendrons of these NPs have a 3,5disubstituted structure, whereas the rest of dendrons (L2, L3, L9) have a wedge or fan shape. In this case, the isothermal treatment also increases the size and shape homogeneity (Table 2 ). For this compound, the best results (in growth and homogeneity) of the thermal treatment are obtained with very short thermal treatments of around $30 \mathrm{~min}$ (Table 2).

The electron microscope can be easily switched from the transmission mode to the diffraction mode, and this allows recording selected area electron-diffraction (SAED) patterns. These patterns indicate that the NPs contain gold crystallized in a fcc phase and that this phase evolves from a more amorphous situation at $t=0 \mathrm{~min}$ to a situation with a higher crystallinity degree, as revealed by the sharpening of the diffraction maxima (see the example in Table S7 and Figures S14 and S15 in Section 3.4 of the Supporting Information).

The thermally tuned materials were optically characterized by UV-vis spectroscopy. Most of the treated AuNPs show RSP bands that extend from 509 to $535 \mathrm{~nm}$, produced by the interaction between the electromagnetic radiation and the electron fluctuation on the surface of the NPs (see Table 2). As expected, for each type of NPs, the $\lambda_{\max }$ of the plasmonic bands shifts to higher wavelengths upon increasing the NP size. However, as can be seen in Table 2, the influence of the ligand is also important when comparing NPs of similar size. Figure $5 \mathrm{k}$ shows the evolution of the RSP band for AuDTL2. It can be observed that, for this compound, the RSP band is visible after $60 \mathrm{~min}$ and its intensity and sharpness increase upon increasing the reaction time. This phenomenon is observed for all the AuDTL $n$ NPs that show a mean diameter bigger than $3.5 \mathrm{~nm}$ and is related to the larger diameters and reduced dispersity (see Table 2). The exception to this general behavior is AuDTL3 that shows a RSP band even in NPs with only $2.4 \mathrm{~nm}$ (treated for 15 $\mathrm{min}$ ) and $2.7 \mathrm{~nm}$ (treated for $30 \mathrm{~min}$ ) of mean diameter. This particular behavior can be attributed to the existence of some NPs of bigger size in these samples.

\section{CONCLUSIONS}

A new strategy has been successfully developed to achieve controlled growth of DenNPs by a simple process under mild conditions. In the first stage of this process, dendritic ligands, bearing a long flexible chain in the focal point, have been incorporated by ligand exchange reactions using DCM as the solvent. During the reaction and purification operations, no aggregation or decomposition processes of the molecules have been observed. Hybrid nanomaterials have been carefully characterized and it is deduced that ligand exchange in the NPs is not total and that the exchange process increases the total number of ligands in the NPs. The magnitude of these changes is related to the features of the dendronic structures.

Subsequently, the hybrid nanomaterials have been thermally treated under mild conditions in the absence of solvent. The magnitude of the growth of the metallic cores and their size distribution depend on the chemical structure of the ligands and the functionalization degree after the ligand-exchange reaction. We have proposed a mechanism that involves the start of the thermal process with the seed formation by thermal shock and, after that, the continuous growth of the cores by slow diffusion through the organic dendritic medium. The combination of both factors, diffusion through the dendritic matrices and the temperature, causes certain fluidity in the medium, and no decomposition of the ligands is observed. After comparing the previous methodologies with our results, our methodology does not imply the destruction of ligands during heat treatment or the use of solvents of any kind to control the size and shape of the NPs. We believe that this approach offers some advantages over similar techniques as it can be considered an efficient and environmentally friendly process to guide the progressive and controlled growth of these metal centers.

\section{ASSOCIATED CONTENT}

\section{Supporting Information}

The Supporting Information is available free of charge at https://pubs.acs.org/doi/10.1021/acsomega.0c04662.

Materials and methods and experimental procedures for the synthesis and characterization of the AuDT and AuDTLn NPs, their organic precursors, and the treated NPs (NMR, FTIR; MS, XPS, STEM, TGA, SAED) (PDF)

\section{AUTHOR INFORMATION}

\section{Corresponding Author}

José Luis Serrano - Instituto de Nanociencia y Materiales de Aragón (INMA), Departamento de Química Orgánica, Universidad de Zaragoza-CSIC, 50009 Zaragoza, Spain; ○ orcid.org/0000-0001-9866-6633; Email: joseluis@ unizar.es

\section{Authors}

José Antonio Ulloa - Instituto de Nanociencia y Materiales de Aragón (INMA), Departamento de Química Orgánica, Universidad de Zaragoza-CSIC, 50009 Zaragoza, Spain; Departamento de Química Orgánica, Facultad de Ciencias Químicas, Universidad de Concepción, Casilla 160-C Concepción, Chile

Joaquín Barberá - Instituto de Nanociencia y Materiales de Aragón (INMA), Departamento de Química Orgánica, Universidad de Zaragoza-CSIC, 50009 Zaragoza, Spain; (ㄱ) orcid.org/0000-0001-5816-7960

Complete contact information is available at: https://pubs.acs.org/10.1021/acsomega.0c04662

\section{Author Contributions}

The manuscript was written through contributions of all authors. All authors have given approval to the final version of the manuscript.

\section{Notes}

The authors declare no competing financial interest.

\section{ACKNOWLEDGMENTS}

This work was supported by the MINECO-FEDER funds (project PGC2018-097583-B-I00) and Gobierno de AragónFSE (Research Group E47_20R). The authors acknowledge the use of SAI (UZ), CEQMA-(UZ-CSIC), and LMA (UZ-CSIC) services. J. A. Ulloa thanks the Becas-Chile program of the 
National Commission for Scientific and Technological Research (CONICYT, Chile) for his PhD grant.

\section{REFERENCES}

(1) Madkour, L. Why So Much Interest in Nanomaterials (NMs)?. In Nanoelectronic Materials: Fundamentals and Applications; Mad-kour, L., Ed.; Springer Nature Switzerland: Basel, 2019; pp 123-140.

(2) Jeevanandam, J.; Barhoum, A.; Chan, Y. S.; Dufresne, A.; Danquah, M. K. Review on nanoparticles and nanostructured materials: history, sources, toxicity and regulations. Beilstein J. Nanotechnol. 2018, 9, 1050-1074.

(3) Khan, I.; Saeed, K.; Khan, I. Nanoparticles: Properties, applications and toxicities. Arabian J. Chem. 2017, 7, 908-931.

(4) Kolwas, K. Decay Dynamics of Localized Surface Plasmons: Damping of Coherences and Populations of the Oscillatory Plasmon Modes. Plasmonics 2019, 14, 1629-1637.

(5) Burunkova, J. A.; Denisiuk, I. Y.; Zhuk, D. I.; Daroczi, L.; Csik, A.; Csarnovics, I.; Kokenyesi, S. Fabrication and properties of luminescence polymer composites with erbium/ytterbium oxides and gold nanoparticles. Beilstein J. Nanotechnol. 2016, 7, 630-636.

(6) Feng, A. L.; You, M. L.; Tian, L.; Singamaneni, S.; Liu, M.; Duan, Z.; Lu, T. J.; Xu, F.; Lin, M. Distance-Dependent Plasmon-Enhanced Fluorescence of Upconversion Nanoparticles using Poly-electrolyte Multilayers as Tunable Spacers. Sci. Rep. 2015, 5, 7779.

(7) Li, N.; Zhao, P.; Astruc, D. Anisotropic Gold Nanoparticles: Synthesis, Properties, Applications, and Toxicity. Angew. Chem., Int. Ed. 2014, 53, 1756-1789.

(8) Fortes Brollo, M. E.; Hernández Flores, P.; Gutiérrez, L.; Johansson, C.; Barber, D. F.; Morales, M. d. P. Magnetic properties of nanoparticles as a function of their spatial distribution on liposomes and cells. Phys. Chem. Chem. Phys. 2018, 20, 17829-17838.

(9) Qi, M.; Zhang, K.; Li, S.; Wu, J.; Pham-Huy, C.; Diao, X.; Xiao, D.; $\mathrm{He}, \mathrm{H}$. Superparamagnetic $\mathrm{Fe} 3 \mathrm{O} 4$ nanoparticles: synthesis by a solvothermal process and functionalization for a magnetic targeted curcumin delivery system. New J. Chem. 2016, 40, 4480-4491.

(10) Nealon, G. L.; Donnio, B.; Greget, R.; Kappler, J.-P.; Terazzi, E.; Gallani, J.-L. Magnetism in gold nanoparticles. Nanoscale 2012, 4, 5244-5258.

(11) Kay, E. R. Dynamic Covalent Nanoparticle Building Blocks. Chem.-Eur. J. 2016, 22, 10706-10716.

(12) Zhang, J.; Mou, L.; Jiang, X. Surface chemistry of gold nanoparticles for health-related applications. Chem. Sci. 2020, 11, 923-936.

(13) Boles, M. A.; Ling, D.; Hyeon, T.; Talapin, D. V. The surface science of nanocrystals. Nat. Mater. 2016, 15, 141-153.

(14) Yamamoto, K.; Imaoka, T.; Tanabe, M.; Kambe, T. New Horizon of Nanoparticle and Cluster Catalysis with Dendrimers. Chem. Rev. 2020, 120, 1397-1437.

(15) Kim, K.; Lee, J.; Jo, G.; Shin, S.; Kim, J.-B.; Jang, J.-H. Dendrimer-Capped Gold Nanoparticles for Highly Reliable and Robust Surface Enhanced Raman Scattering. ACS Appl. Mater. Interfaces 2016, 8, 20379-20384.

(16) Wang, X.; Wang, H.; Wang, Y.; Yu, X.; Zhang, S.; Zhang, Q.; Cheng, Y. A Facile Strategy to Prepare Dendrimer-stabilized Gold Nanorods with Sub-10-nm Size for Efficient Photothermal Cancer Therapy. Sci. Rep. 2016, 6, 22764.

(17) Kanie, K.; Matsubara, M.; Zeng, X.; Liu, F.; Ungar, G.; Nakamura, H.; Muramatsu, A. Simple Cubic Packing of Gold Nanoparticles through Rational Design of Their Dendrimeric Corona. J. Am. Chem. Soc. 2012, 134, 808-811.

(18) Garcia-Martinez, J. C.; Crooks, R. M. Extraction of Au Nanoparticles Having Narrow Size Distributions from within Dendrimer Templates. J. Am. Chem. Soc. 2004, 126, 16170-16178.

(19) Kabachii, Y. A.; Golub, A. S.; Kochev, S. Y.; Lenenko, N. D.; Abramchuk, S. S.; Antipin, M. Y.; Valetsky, P. M.; Stein, B. D.; Mahmoud, W. E.; Al-Ghamdi, A. A.; Bronstein, L. M. Multifunctional Nanohybrids by Self-Assembly of Monodisperse Iron Oxide Nanoparticles and Nanolamellar MoS2 Plates. Chem. Mater. 2013, 25, 24342440.
(20) Daniel, M.-C.; Astruc, D. Gold Nanoparticles: Assembly, Supramolecular Chemistry, Quantum-Size-Related Properties, and Applications toward Biology, Catalysis, and Nanotechnology. Chem. Rev. 2004, 104, 293-346.

(21) Godipas, K. R.; Whitesell, J. K.; Fox, M. A. Nanoparticles- Cored Dendrimers: Synthesis and Characterization. J. Am. Chem. Soc. 2003, $125,6491-6502$.

(22) Donnio, B.; García-Vázquez, P.; Gallani, J.-L.; Guillon, D.; Terazzi, E. Dendronized Ferromagnetic Gold Nanoparticles SelfOrganized in a Thermotropic Cubic Phase. Adv. Mater. 2007, 19, 3534-3539.

(23) Advincula, R. C. Hybrid organic-inorganic nanomaterials based on polythiophene dendronized nanoparticles. Dalton Trans. 2006, $2728-2784$.

(24) Liu, Y.; Kim, M.; Wang, Y.; Wang, Y. A.; Peng, X. Higly Luminescent, Stable, and Water-Soluble CdSe/CdS Core-Shell Dendron Nanocrystals with Carboxylate Anchoring Groups. Langmuir 2006, $22,6341-6345$

(25) Elbert, K. C.; Jishkariani, D.; Wu, Y.; Lee, J. D.; Donnio, B.; Murray, C. B. Design, Self-Assembly, and Switchable Wettability in Hydrophobic, Hydrophilic, and Janus Dendritic Ligand-Gold Nanoparticle Hybrid Materials. Chem. Mater. 2017, 29, 8737-8746.

(26) Martí Coma-Cros, E.; Lancelot, A.; San Anselmo, M.; Neves Borgheti-Cardoso, L.; Valle-Delgado, J. J.; Serrano, J. L.; FernàndezBusquets, X.; Sierra, T. Micelle carriers based on dendritic macromolecules containing bis-MPA and glycine for antimalarial drug delivery. Biomater. Sci. 2019, 7, 1661-1674.

(27) Fedeli, E.; Lancelot, A.; Dominguez, J.; Serrano, J.; Calvo, P.; Sierra, T. Self-Assembling Hybrid Linear-Dendritic Block Copolymers: The Design of Nano-Carriers for Lipophilic Antitumoral Drugs. Nanomaterials 2019, 9, 161.

(28) Moscariello, P.; Ng, D. Y. W.; Jansen, M.; Weil, T.; Luhmann, H. J.; Hedrich, J. Brain Delivery of Multifunctional Dendrimer Protein Bioconjugates. Adv. Sci. 2018, 5, 1700897.

(29) Garrigue, P.; Tang, J.; Ding, L.; Bouhlel, A.; Tintaru, A.; Laurini, E.; Huang, Y.; Lyu, Z.; Zhang, M.; Fernandez, S.; Balasse, L.; Lan, W.; Mas, E.; Marson, D.; Weng, Y.; Liu, X.; Giorgio, S.; Iovanna, J.; Pricl, S.; Guillet, B.; Peng, L. Self-assembling supramolecular dendrimer nanosystem for PET imaging of tumors. Proc. Natl. Acad. Sci. U.S.A. 2018, 115, 11454-11459.

(30) Movellan, J.; Urbán, P.; Moles, E.; de la Fuente, J. M.; Sierra, T.; Serrano, J. L.; Fernàndez-Busquets, X. Amphiphilic dendritic derivatives as nanocarriers for the targeted delivery of antimalarial drugs. Biomaterials 2014, 35, 7940-7950.

(31) Concellón, A.; Termine, R.; Golemme, A.; Romero, P.; Marcos, M.; Serrano, J. L. High hole mobility and light-harvesting in discotic nematic dendrimers prepared via 'click' chemistry. J. Mater. Chem. C 2019, 7, 2911-2918.

(32) Iguarbe, V.; Concellón, A.; Termine, R.; Golemme, A.; Barberá, J.; Serrano, J. L. Making Coaxial Wires Out of Janus Den-drimers for Efficient Charge Transport. ACS Macro Lett. 2018, 7, 1138-1143.

(33) Concellón, A.; Liang, T.; Schenning, A. P. H. J.; Serrano, J. L.; Romero, P.; Marcos, M. Proton-conductive materials formed by coumarin photocrosslinked ionic liquid crystal dendrimers. J. Mater. Chem. C 2018, 6, 1000-1007.

(34) Concellón, A.; Marcos, M.; Romero, P.; Serrano, J. L.; Termine, R.; Golemme, A. Not Only Columns: High Hole Mobility in a Discotic Nematic Mesophase Formed by Metal-Containing Porphy-rin-Core Dendrimers. Angew. Chem., Int. Ed. 2017, 56, 1259-1263.

(35) Abd-El-Aziz, A. S.; Abdelghani, A. A.; Wagner, B. D.; Abdelrehim, E. M. Aggregation enhanced excimer emission (AEEE) with efficient blue emission based on pyrene dendrimers. Polym. Chem. 2016, 7, 3277-3299.

(36) Si, K. J.; Chen, Y.; Shi, Q.; Cheng, W. Nanoparticle Superlattices: The Roles of Soft Ligands. Adv. Sci. 2017, 5, 1700179.

(37) Jishkariani, D.; Diroll, B. T.; Cargnello, M.; Klein, D. R.; Hough, L. A.; Murray, C. B.; Donnio, B. Dendron-Mediated Engi-neering of Interparticle Separation and Self-Assembly in Dendronized Gold 
Nanoparticles Superlattices. J. Am. Chem. Soc. 2015, 137, 1072810734.

(38) Lewandowski, W.; Fruhnert, M.; Mieczkowski, J.; Rockstuhl, C.; Górecka, E. Dynamically self-assembled silver nanoparticles as a thermally tunable metamaterial. Nat. Commun. 2015, 6, 6590.

(39) Van Gough, D.; Juhl, A. T.; Braun, P. V. Programming struc-ture into 3D nanomaterials. Mater. Today 2009, 12, 28-35.

(40) Zacharaki, E.; Kalyva, M.; FjellvaÅg, H.; SjaÅstad, A. O. Burst nucleation by hot injection for size controlled synthesis of $\varepsilon$-cobalt nanoparticles. Chem. Cent. J. 2016, 10, 10.

(41) Jansons, A. W.; Hutchison, J. E. Continuous Growth of Metal Oxide Nanocrystals: Enhanced Control of Nanocrystal Size and Radial Dopant Distribution. ACS Nano 2016, 10, 6942-6951.

(42) Williams, J. V.; Kotov, N. A.; Savage, P. E. A Rapid Hot-Injection Method for the Improved Hydrothermal Synthesis of CdSe Nanoparticles. Ind. Eng. Chem. Res. 2009, 48, 4316-4321.

(43) de Mello Donegá, C.; Liljeroth, P.; Vanmaekelbergh, D. Physicochemical Evaluation of the Hot-Injection Method, a Synthesis Route for Monodisperse Nanocrystals. Small 2005, 1, 1152-1162.

(44) Ranjbar, M.; Yousefi, M. Synthesis and Characterization of Lanthanum Oxide Nanoparticles from Thermolysis of Nano-sized Lanthanum(III) Supramolecule as a Novel Precursor. J. Inorg. Organomet. Polym. Mater. 2014, 24, 652-655.

(45) Tuchscherer, A.; Schaarschmidt, D.; Schulze, S.; Hietschold, M.; Lang, H. Gold nanoparticles generated by thermolysis of "all-in-one" gold(i) carboxylate complexes. Dalton Trans. 2012, 41, 2738-2746.

(46) Erk, C.; Eric Yau, M. Y.; Lange, H.; Thomsen, C.; Miclea, P.; Wehrspohn, R. B.; Schlecht, S.; Steinhart, M. Formation of gold nanoparticles in polymeric nanowires by low-temperature thermolysis of gold mesitylene. J. Mater. Chem. 2012, 22, 684-690.

(47) Chen, Y.-B.; Chen, L.; Wu, L.-M. Structure-Controlled Solventless Thermolytic Synthesis of Uniform Silver Nanodisks. Inorg. Chem. 2005, 44, 9817-9822.

(48) Niu, Y.; Yeung, L. K.; Crooks, R. M. Size-selective Hydrogenation of Olefins by Dendrimer-Encapsulatued Palladim Nanoparticles. J. Am. Chem. Soc. 2001, 123, 6840-6846.

(49) Sutton, A.; Franc, G.; Kakkar, A. Silver metal nanoparticles: Facile dendrimer-assisted size-controlled synthesis and selective catalytic reduction of chloronitrobenzenes. J. Polym. Sci., Part A: Polym. Chem. 2009, 47, 4482-4493.

(50) Garzoni, M.; Cheval, N.; Fahmi, A.; Danani, A.; Pavan, G. M. Ionselective Controlled Assembly of Dendrimer-Based Functional Nanofibers and Their Ionic-Competitive Disassembly. J. Am. Chem. Soc. 2012, 134, 3349-3357.

(51) Percec, V.; Cho, W.-D.; Ungar, G.; Yeardley, D. J. P. Synthe-sis and Structural Analysis of Two Constitutional Isomeric Libraries of AB2-Based Monodendrons and Supramolecular Dendrimers. J. Am. Chem. Soc. 2001, 123, 1302-1315.

(52) Percec, V.; Cho, W.-D.; Ungar, G.; Yeardley, D. J. P. From Molecular Flat Tapers, Discs, and Cones to Supramolecular Cylinders and Spheres using Fréchet-Type Monodendrons Modified on their Periphery. Angew. Chem., Int. Ed. 2000, 39, 1597-1602.

(53) Percec, V.; Cho, W.-D.; Ungar, G. Increasing the Diameter of Cylindrical and Spherical Supramolecular Dendrimers by Decreasing the Solid Angle of Their Monodendrons via Periphery Functionalization. J. Am. Chem. Soc. 2000, 122, 10273-10281.

(54) Percec, V.; Cho, W.-D.; Mosier, P. E.; Ungar, G.; Yeardley, D. J. P. Structural Analysis of Cylindrical and Spherical Supramolec-ular Dendrimers Quantifies the Concept of Monodendron Shape Control by Generation Number. J. Am. Chem. Soc. 1998, 120, 11061-11070.

(55) Balagurusamy, V. S. K.; Ungar, G.; Percec, V.; Johansson, G. Rational Design of the First Spherical Supramolecular Dendrimers SelfOrganized in a Novel Thermotropic Cubic Liquid-Crystalline Phase and the Determination of Their Shape by X-ray Analysis. J. Am. Chem. Soc. 1997, 119, 1539-1555.

(56) Frechet, J. M. J. Dendrimers and supramolecular chemistry. Proc. Natl. Acad. Sci. U.S.A. 2002, 99, 4782-4787.
(57) Stadler, A.-M. Structural Features of Fréchet-Type Dendrons and Dendrimers in Single Crystals. Cryst. Growth Des. 2010, 10, 50505065 .

(58) Brust, M.; Walker, M.; Bethell, D.; Schiffrin, D. J.; Whyman, R. Synthesis of thiol-derivatised gold nanoparticles in a two-phase LiquidLiquid system. Chem. Commun. 1994, 801-802.

(59) Hostetler, M. J.; Templeton, A. C.; Murray, R. W. Dynamics of Place-Exchange Reactions on Monolayer-Protected Gold Cluster Molecules. Langmuir 1999, 15, 3782-3789.

(60) Gentry, S. T.; Kendra, S. F.; Bezpalko, M. W. Ostwald Ripening in Metallic Nanoparticles: Stochastic Kinetics. J. Phys. Chem. C 2011, $115,12736-12741$.

(61) Vengrenovich, R. D.; Ivanskii, B. V.; Panko, I. I.; Yarema, S. V.; Kryvetskyi, V. I.; Stasyk, M. O. Ostwald Ripening of the Plati-num Nanoparticles in the Framework of the Modified LSW Theory. J. Nanomater. 2014, 2014, 821584.

(62) Gommes, C. J. Ostwald ripening of confined nanoparticles: chemomechanical coupling in nanopores. Nanoscale 2019, 11, 73867393. 